\title{
A Psicologia em Publicações Científicas: Um Estudo Histórico no Periódico
}

\author{
“Ciência e Cultura"(1949-1969)
}

\author{
Felipe Maciel dos Santos Souza* \\ Universidade Federal da Grande Dourados - UFGD, Dourados, MS, Brasil \\ ORCID: http://orcid.org/0000-0002-2282-7784 \\ José Alberto Lechuga de Andrade Filho*** \\ Universidade Federal de Mato Grosso do Sul - UFMS, Campo Grande, MS, Brasil \\ ORCID: http://orcid.org/0000-0002-0311-3372 \\ Rodrigo Lopes Miranda*** \\ Universidade Católica Dom Bosco - UCDB, Campo Grande, MS, Brasil \\ ORCID: http://orcid.org/0000-0003-3222-7368
}

\begin{abstract}
RESUMO
A formação e a profissão de psicólogo no Brasil foram regulamentas em 1962, com a promulgação da Lei n. 4119. Entretanto, a tramitação ocorreu por aproximadamente dez anos, em decorrência de debates e tensões entre diferentes atores sociais envolvidos com a área. Esta pesquisa objetivou descrever e analisar produções vinculadas à Psicologia, publicadas em um dos principais periódicos científicos brasileiros à época, Ciência e Cultura, no entorno temporal da promulgação da referida Lei (1949-1969). A pesquisa caracteriza-se como História do Tempo Presente, ancorada na História da Psicologia e na História das Ciências. O periódico analisado possui uma seção de Psicologia desde seu primeiro número (1949) em que observamos constantes publicações que, após a regulamentação da profissão, sofreram um aumento. Além disto, o número de artigos publicados sobre Psicologia é muito menor se comparado ao número de resumos científicos. Entre os autores, identificamos uma leve predominância do gênero feminino. Quanto às vinculações institucionais, verificamos uma alta incidência de materiais produzidos na região Sudeste. Foi possível identificar e analisar o que era produzido sobre Psicologia, e que aparecia em um influente vetor de circulação da ciência brasileira, no período.
\end{abstract}

Palavras-chave: história da psicologia, divulgação científica, regulamentação profissional.

\section{Psychology in Scientific Publications: A Historical Study in the Journal}

\section{Ciência e Cultura (1949-1969)}

ABSTRACT
In 1962, the training and profession of psychologist were legislated in Brazil from the
establishment of the Law $4.119 / 62$. However, this process lasted for approximately ten years
\begin{tabular}{|l|l|l|l|l|l|}
\hline Estudos e Pesquisas em Psicologia & Rio de Janeiro & v. 21 & n. 1 & p. $357-378$ & $\begin{array}{c}\text { Janeiro a Abril } \\
\text { de } 2021\end{array}$ \\
\hline
\end{tabular}


because of controversies between different actors involved with the area. This research describes and analyzes texts linked to Psychology published in Ciência e Cultura, one of the main Brazilian scientific journals, at the time. The research timeframe comprehends the 1950's and the 1960's, period of the legalization aforementioned. It is characterized as History of Present Time, anchored in the History of Psychology and History of Science. Ciência e Cultura has a Psychology section since its first number (1949) in which there are constant publications whose frequency increased after the establishment of the training and profession of psychologist, in the country. In addition to this, the number of published articles on Psychology is much smaller compared to the number of its abstracts. Among the authors, there was a slight predominance of the female gender, and considering the institutional affiliations, there was a high incidence of manuscripts from the Southeast region of Brazil. Therefore, it was possible to identify and analyze what was produced and had circulated, on Psychology, in the country, as a vector of Brazilian science in the period.

Keywords: history of psychology, scientific popularization, professional, regulation.

\section{Psicología en Publicaciones Científicas: Un Estudio Histórico en la Revista}

\section{Ciência e Cultura (1949-1969)}

\section{RESUMEN}

La formación y la profesión de psicólogo en Brasil fueron reguladas en 1962, con la promulgación de la Ley n. 4119. Sin embargo, su tramitación ha ocurrido durante aproximadamente diez años, debido a debates y tensiones entre los diferentes actores sociales involucrados en el ámbito. Esta investigación tuvo como objetivo describir y analizar producciones vinculadas a la psicología, publicadas en una de las principales revistas científicas brasileñas en aquella época, Ciência e Cultura, en el contexto temporal de la promulgación de dicha Ley (1949-1969). La investigación se caracteriza como Historia del Tiempo Presente, anclada en la Historia de la Psicología y en la Historia de las Ciencias. La revista analizada tiene una sección de Psicología desde su primer número (1949) en que observamos que, después de la regulación de la profesión, publicaciones constantes presentaron un aumento. Además, el número de artículos publicados sobre psicología es mucho menor en comparación con el número de resúmenes científicos. Entre los autores, identificamos una ligera predominancia del género femenino. Con respecto a las vinculaciones institucionales, encontramos una alta incidencia de materiales producidos en la región Sudeste. Fue posible identificar y analizar lo que se produjo acerca de la psicología, y que aparecía en un influyente vector de circulación de la ciencia brasileña, en aquel período.

Palabras clave: historia de la psicología, divulgación científica, reglamentación profesional.

Contemporaneamente, tem ocorrido debates relacionados a diversos aspectos da Psicologia, tais como o acesso a métodos e técnicas particulares, a definição de seus objetos de trabalho, a caracterização de suas funções privativas, a delimitação de seu campo de 
trabalho, dentre outros. Nesse cenário, vemos tensões que se materializam em projetos de lei e debates legais, a exemplo do Projeto de Lei n. 4931/2016 (Teixeira, 2016), popularmente conhecido como "cura gay", e a Lei n. 12.842 (2013), também denominada "Lei do Ato Médico”. Esse conjunto impacta, ainda, discussões sobre a formação do psicólogo, particularmente no momento de debates sobre as novas Diretrizes Curriculares Nacionais (DCNs) que vem se desenvolvendo desde o início de 2019. Dessa forma, os diferentes atores relacionados às práticas da Psicologia, na atualidade, têm de se haver com questões diversas: que profissionais se quer formar? Quais são os objetos de trabalho da Psicologia? Quais são as funções privativas deste profissional? Como se relacionar com outras profissões da saúde? E muitas outras. Respostas a tais perguntas impactam não apenas a comunidade de psicólogos, mas, também, o público atendido por tais profissionais.

Questões como aquelas convidam diferentes possibilidades de pesquisa e, dentre elas, pesquisas historiográficas (Mota, Cara, \& Miranda, 2019). Entende-se, para tanto, que o historiador - neste caso, um historiador da Psicologia - tem um compromisso com o presente ao perscrutar o passado (Ariès, 1978/2005; Brock, 2015). Ou seja, para se interrogar o passado, (i) partimos de uma questão presente para, então, operarmos a investigação no regime de historicidade do fenômeno pesquisado e, então, (ii) (re)construírmos tal história a partir de um compromisso em torná-la inteligível para a audiência contemporânea. Assim, aquelas questões atuais sobre a formação e a atuação do psicólogo, no presente, têm convidado a um exame do contexto de produção de sua formação e profissão, no Brasil e alhures (Buchanan, 2003; Castro \& Alcântara, 2011; Klappenbach, 2000; Rudá, Coutinho, \& Almeida Filho, 2015). No contexto brasileiro, tais investigações nos convidam, dentre outras possibilidades, a um exame da história da Lei n. 4.119/62 - que regulamenta a formação e o exercício profissional da Psicologia, no país - e das legislações que estabelecem o Sistema Conselhos- Conselho Federal de Psicologia (CFP) e Conselhos Regionais (CRs). Isso ocorre porque tal literatura nos sinaliza que a conformação de tais fenômenos ocorreu em quadro de tensionamentos e aproximações entre diferentes atores sociais que, antes da regulamentação, pesquisavam e atuavam no campo da Psicologia. Dessa maneira, investigações com visadas para o passado podem nos ajudar a compreender certos cortes e continuidades em práticas científicas, profissionais e legais envolvidas na conformação da Psicologia. A compressão de tais aspectos, por sua vez, nos permitiria uma leitura crítica das problemáticas presentes.

Diante disso, este texto tem por objetivo descrever e analisar produções vinculadas à Psicologia, veiculadas no periódico "Ciência e Cultura", entre 1949 e 1969. O periódico foi escolhido por ter sido uma das primeiras revistas cientificas brasileiras - criada em 1949 pela 
Sociedade Brasileira para o Progresso da Ciência (SBPC) e ainda em circulação. Nossa hipótese é que haveria publicações em Psicologia no referido periódico para além daquelas que ocorriam em revistas vinculadas diretamente ao campo Psi. Neste cenário, à época, havia os Arquivos Brasileiros de Psicotécnica (ABP) - editorado pelo Instituto de Seleção e Orientação Profissional (ISOP), vinculado à Fundação Getúlio Vargas (FGV) no Rio de Janeiro - e o Boletim de Psicologia - editorado pela Associação de Psicologia de São Paulo -, ambos criados em 1949. Assim, acessar aquelas produções em recorte temporal que compreende os debates sobre a formação e a profissão de Psicologia, no país, bem como o estabelecimento do Sistema Conselhos, auxiliaria a lançar uma luz historiográfica sobre aqueles debates. Metodologicamente, esta pesquisa se ancora em uma História Social da Psicologia (Jansz \& van Drunen, 2004) e na História Crítica da Psicologia (Danziger, 1985), conformando-se como uma investigação sociobibliométrica (Klappenbach, 2017; Millán, Polanco, Ossa, Béria, \& Cudina, 2017).

\section{Método}

De acordo com as definições de Montero e León (2007), esta investigação historiográfica se caracteriza como ex post facto, i.e., tem por objetivo compreender relações entre um determinado fato e um fenômeno que ocorre a posteriori. Nesta perspectiva, parte-se de um plano retrospectivo de correlação, qual seja: sabemos que psicólogos (i) pesquisavam e atuavam no Brasil antes da criação de cursos de graduação em Psicologia, bem como da regulamentação da formação e da profissão; (ii) esses mesmos atores sociais estiveram envolvidos nos debates da Lei n. 4.119/62 e do Sistema Conselhos e (iii) parte daquelas atividades de pesquisa e intervenção eram tornadas públicas no periódico "Ciência e Cultura". Assim, assume-se que a ciência em circulação em tal periódico pode nos ajudar a compreender certo cenário intelectual quando dos debates legais da formação e profissão, no país. Este delineamento de estudo tem sido levado a cabo em estudos similares no cenário nacional (Mota, Castro Neto, \& Miranda, 2016; Mota \& Miranda, 2017; Xavier \& Miranda, 2018) e internacional (Fernandez, Polanco, Pereira, Beria, \& Zapico, 2017; Polanco, Beria, \& Klappenbach, 2017) da História da Psicologia.

A equipe de pesquisa foi composta por três pesquisadores. Dois deles fizeram a busca das fontes primárias entre os dias 12 de novembro de 2018 e 17 de dezembro do mesmo ano, no sítio eletrônico da Biblioteca Nacional, na seção da Hemeroteca. Na seção identificada, foi selecionado o periódico analisado e delimitado o período de busca, 1949 a 1969. Os termos de 
busca foram: psicologia, psicologista, psicotécnico(a) e psicometria. Tais termos foram utilizados considerando que, à época, estavam associados ao fazer da Psicologia e à sua potencial nomenclatura. Portanto, seriam termos que aproximariam a equipe de textos vinculados à Psicologia. Após a busca realizada separadamente por cada pesquisador, houve comparação dos resultados, produzindo-se uma listagem final de análise que, por sua vez, foi avaliada pelo terceiro membro da equipe. Após a concordância dos três investigadores, foi iniciada a descrição e análise do material. Para o tratamento e análise de dados foi utilizado o programa Microsoft Excel, versão 2010.

\section{Procedimentos}

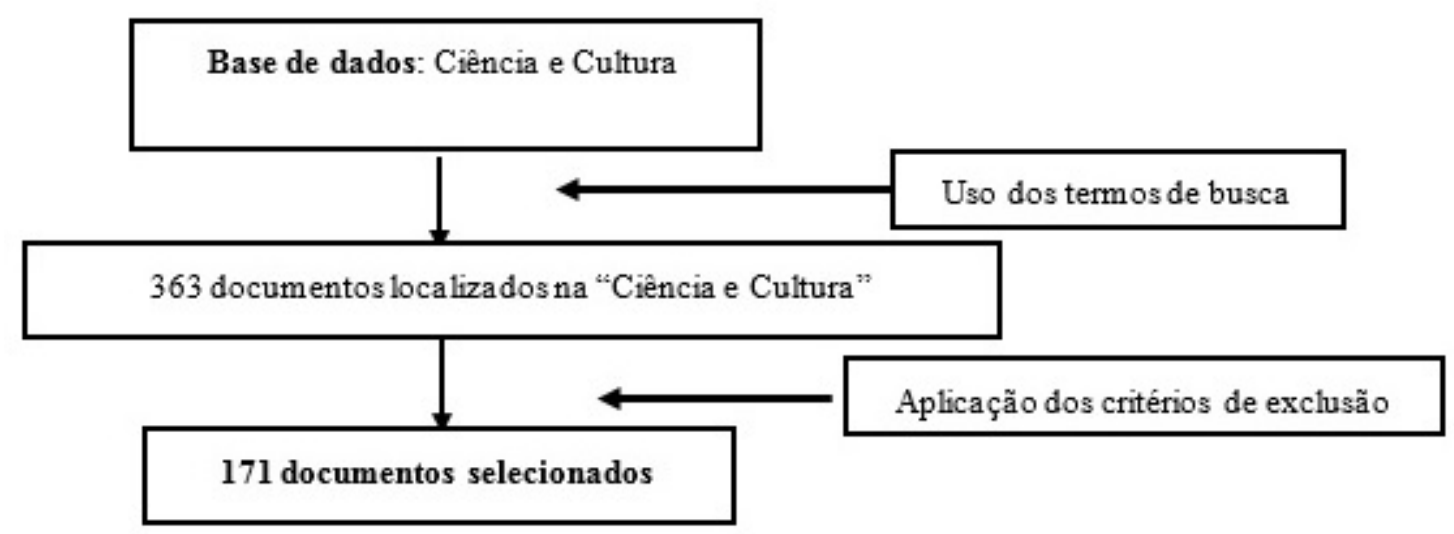

Figura 1. Fluxograma do processo de seleção dos documentos

A Figura 1 sumariza os procedimentos de busca e a aplicação dos critérios de inclusão e exclusão amostral. Assim, a partir do uso dos termos de busca, nos títulos e corpo do texto, foram registrados 363 documentos. Todos os títulos e textos foram lidos pela equipe da pesquisa e foram aplicados os seguintes critérios de exclusão, a fim de excluirmos os materiais que não estavam de acordo com os objetivos da pesquisa: não ser relato de pesquisa; não abordar temas de Psicologia ou áreas temáticas de Psicologia. Assim, compuseram o corpus documental desta pesquisa 171 textos que foram lidos na íntegra e cujo conteúdo foi analisado. 


\section{Resultados e Discussão}

Apesar de termos pesquisas e a criação de instituições científicas, no Brasil, desde o início do século XIX, foi a partir das primeiras décadas do século XX que ocorreu uma maior sistematização da ciência nacional (Dantes, 2001; Sá, 2006). Isso pode ser observado pela criação tanto de universidades quanto de sociedades científicas e agências de fomento. Por exemplo, foi criada, em 1920, a Universidade do Rio de Janeiro, atualmente Universidade Federal do Rio de Janeiro (UFRJ); em 1927, a Universidade de Minas Gerais (atualmente, Universidade Federal de Minas Gerais [UFMG]) e, em 1934, a Universidade de São Paulo (USP). No que tange às sociedades científicas, temos os exemplos da Sociedade Brasileira de Ciências (atualmente, Academia Brasileira de Ciências [ABC]), instituída em 1916 e da SBPC, criada em 1948. Houve, ainda, a criação de agências de fomento, tais como do Conselho Nacional de Desenvolvimento Científico e Tecnológico $(\mathrm{CNPq})$ e da Coordenação de Aperfeiçoamento de Pessoal de Nível Superior (CAPES), em 1951. Este período coincide, também, com iniciativas de institucionalização da Psicologia brasileira por meio da criação de Institutos e da regulamentação de um currículo mínimo e do campo profissional.

Em 1932, o laboratório de Psicologia da Colônia de Psicopatas, em Engenho de Dentro, no Rio de Janeiro, foi transformado em Instituto de Psicologia cujo objetivo, dentre outros, era "formar psicólogos profissionais" (Decreto n. 21.173, 1932). Entretanto, este curso ficou em funcionamento por apenas alguns meses, em decorrência de variadas controvérsias entre diferentes atores sociais, tais como seu diretor Waclaw Radecki, o Estado e a Igreja (cf. Centofanti, 1982). A justificativa do Governo Federal para a criação do referido curso era: “[o fato de que] as técnicas psicológicas vêm apresentando, dia a dia, novas e importantes aplicações, na organização racional do trabalho, na orientação e seleção profissionais, na medicina, no direito e na educação.”. Estas aplicações da Psicologia se fortaleceram ao longo da primeira metade do século XX, em função, dentre outros motivos, da criação de instituições, e.g., o Instituto de Seleção e Orientação Profissional (ISOP). Como consequência, a partir de 1949 os debates sobre a formação de psicólogos, no país, ganharam novo ímpeto e, no início da década de 1950, implicou na criação de cursos de graduação (Pontifícia Universidade Católica do Rio de Janeiro [PUC-RJ] em 1953 e Pontifícia Universidade Católica do Rio Grande do Sul [PUC-RS]) em 1954, e da apresentação de um anteprojeto de lei para regulamentação da profissão e formação, em Psicologia, no país em 1954. 
Neste cenário é que se situa a criação, em 1949, da revista "Ciência e Cultura". O periódico tinha como objetivo "difundir não só os conhecimentos que a Ciência vai acumulando, mas também os dados relativos à projeção desses conhecimentos na sociedade" (Sociedade Brasileira para o Progresso da Ciência [SBPC], 1949, p. 3). Em nosso entendimento, sua criação tinha íntima relação com os objetivos da própria SBPC, pois seriam:

(a) a justificação da ciência, mostrando ao público seus progressos, seus métodos de trabalho, suas aplicações e até mesmo suas limitações (...) (b) robustecimento da organização científica nacional, pela melhor articulação dos cientistas (...) (c) luta pela manutenção de elevados padrões de conduta científica, e ao mesmo tempo combate à pseudo e à meia ciência, que tantas vezes tomam posições que deveriam pertencer à verdadeira ciência (...) (SBPC, 1949, p. 2)

Assim, o periódico poderia apresentar "ao público científico e a todos os que se interessam pelos problemas da Ciência" (SBPC, 1949, p. 3) os seus "progressos", como uma forma tanto (i) de organizar a comunidade científica nacional, em consolidação (Schwartzman, 2002), quanto (ii) de produzir espaços sociais para sua promoção como portavozes da modernização e do progresso, pedras angulares de discursos do período (Fausto, 1994/2003; Schwarcz \& Starling, 2015). 


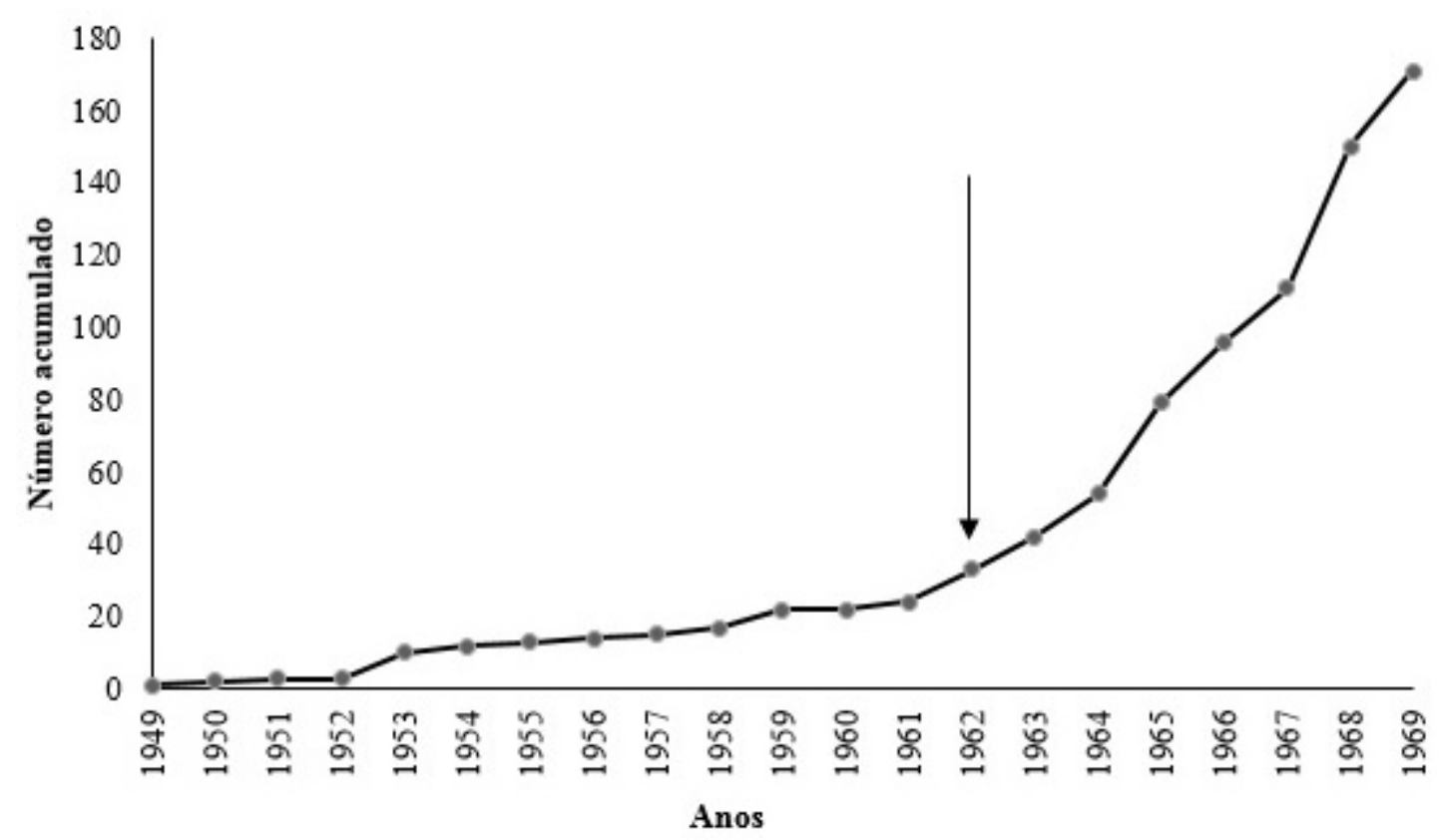

Figura 2. Número acumulado de materiais sobre Psicologia publicados entre 1949 e 1969 no periódico Ciência e Cultura. A seta indica o ano em que foi publicada a Lei $n^{\circ} 4.119$.

Ao longo dos 20 anos analisados nesta pesquisa, contabilizamos 363 textos publicados na "Ciência e Cultura", de diferentes gêneros, tais como artigos e ensaios científicos, críticas, notícias, reportagens e resumos de estudos. Neste montante, os 171 textos indexados como fontes primárias desta pesquisa perfazem apenas 47,10\%, cuja frequência acumulada, entre 1949 e 1969, é vista na Figura 2. Em sua distribuição, vemos que 20 deles foram artigos e 151 foram resumos de investigações apresentadas nos encontros da SBPC. Do total de artigos analisados, constatamos que quatro foram apresentados em encontros da SBPC. Tomando a Figura 1, notamos que desde o primeiro ano da revista houve trabalhos vinculados à Psicologia. A partir de 1953, a curva tende ao aumento gradual com leve plateau entre 1959 e 1960 e, a partir de 1962, houve nítida aceleração nas publicações de Psicologia. Os anos de 1953 e 1962 nos parecem paradigmáticos para a compreensão histórica que ora se apresenta: (i) como dito, foi o período em que foram abertos os primeiros cursos de graduação em Psicologia, na PUC-RJ e PUC-RS, Universidade de São Paulo (USP), Universidade Católica de Minas Gerais (UCMG) e Universidade Católica de Pernambuco (UNICAP), foi submetido para apreciação o Anteprojeto sobre a formação e a profissão de psicóloga, e (ii) em 1962 foi aprovada a Lei n. 4.119.

Entretanto, notamos que aquele aumento nas publicações não esteve relacionado ao aumento do número de artigos, mas do número de resumos veiculados no periódico. Este movimento, conforme exposto na Figura 2, se acentuou na década de 1960, o que pode ter 
relação com o aumento do número de cursos de graduação em Psicologia, no país, à época (Baptista, 2009; Rudá, Coutinho, \& Almeida Filho, 2015). Todavia, vale lembrar que a diferença entre os números de resumos e artigos publicados pode ser justificada pelo fato de o periódico "Ciência e Cultura" apresentar, no período analisado, as pesquisas divulgadas nos encontros anuais da SBPC, dado que pode indicar o periódico como um bom veículo de divulgação científica e que contribuiu para a institucionalização da ciência em nosso país.

A partir dos documentos analisados, foi possível identificarmos a autoria de 169 textos. Considerando apenas os primeiros autores, identificamos 92 nomes distintos e, deste total, $48(52 \%)$ correspondem ao gênero feminino e $44(48 \%)$ ao masculino. Como a diferença é pequena, que poderia ser compreendida por um desvio padrão de apenas 0,052 , não é possível afirmar que houve prevalência do gênero feminino. Todavia, se considerarmos outros estudos (Mota, Castro Neto, \& Miranda, 2016; Mota, 2017), vemos que havia tendências à feminilização da produção científica na Psicologia brasileira à época. Ou seja, os dados podem sugerir que esta presença feminina estaria ocorrendo pari passu à produção de mecanismos de institucionalização da Psicologia, no Brasil. Desta forma, ela não seria um produto de período ulterior, conforme dados nos sugerem nas décadas de 1970 e 1980 (Rosemberg, 1984; P. Rosas, Rosas, \& Xavier, 1988).

Do total de 92 autores que publicaram os materiais vinculados à Psicologia, seis deles perfazem 6,57\% deste montante, a saber: Aniela Meyer Ginsberg $(n=5)$, Arrigo Leonardo Angelini $(n=15)$, Francisco José Amaral Vieira $(n=10)$, Geraldina Porto Witter $(n=7)$, Odette Lourenção Van Kolck $(n=5)$ e Samuel Pfromm Netto $(n=10)$. Com base em Campos (2001), constatamos que as autoras de gênero feminino mais produtivas eram licenciadas em Pedagogia ou Psicologia, dado que se aproxima do encontrado por Jacó-Vilela, Mello, Ferreira, Messias e Lucas (2009). Aniela Meyer Ginsberg foi uma pesquisadora de destaque por seus estudos sobre raça/etnia no Brasil (Cintrão Forghieri, 2011; Cunha \& Santos, 2014). Odette Lourenção Van Kolck, por sua vez, é reconhecida por suas contribuições para a área de Avaliação Psicológica no Brasil, enfatizando o papel do diagnóstico psicológico (Boccato, 2004) enquanto Geraldina Porto Witter atuou em Psicologia Experimental e, particularmente, na área educacional, desde 1960 (Lomonaco, 2014). Os principais pesquisadores do gênero masculino também eram licenciados em Pedagogia ou Psicologia. Merecem destaque Arrigo Leonardo Angelini e Samuel Pfromm Netto. Como indica Witter (1998), Arrigo Leonardo Angelini contribuiu significativamente para a evolução da Psicologia no Brasil tanto como área de conhecimento, em especial a de Psicologia Educacional, como profissão. Samuel Pfromm Netto, além de contribuir para a mesma área de Arrigo Angelini, destacou-se como 
um pesquisador e profissional ativo em várias áreas, como a clínica, a do trabalho e a da mídia (especialmente, em televisão e rádio) (Lomonaco, 2013). Também merece destaque Francisco José Amaral Vieira, que organizou o Laboratório de Psicologia Experimental e o Laboratório de Neurobiologia da Universidade Federal do Ceará (UFC).

Embora a diferença entre os gêneros dos autores não seja expressiva, é importante salientar a presença das mulheres como promotoras de conhecimento da Psicologia no período do entorno temporal da regulamentação da Lei n. 4.119 (1949-1969), tendo em vista que o crescimento do acesso das mulheres ao Ensino Superior só ocorreu a partir do final dos anos 1930, tomando impulso apenas nas décadas seguintes (Machado,1997; Xavier \& Miranda, 2018).

\section{Tabela 1}

Instituições localizadas com maiores ocorrências de publicação no período de 1949 a 1969.

\begin{tabular}{lcc}
\hline \multicolumn{1}{c}{ Instituição } & Frequência & UF \\
\hline USP, campus de São Paulo & 85 & São Paulo \\
$\begin{array}{l}\text { Pontifícia Universidade Católica de } \\
\text { São Paulo (PUC-SP) }\end{array}$ & 15 & São Paulo \\
Universidade Estadual de São Paulo, & 7 & São Paulo \\
campus de Rio Claro & 5 & São Paulo \\
USP, campus de Ribeirão Preto & & \\
\hline
\end{tabular}

Dos 171 documentos analisados, não foi possível obtermos os nomes das instituições às quais autores de oito materiais estavam vinculados. Assim sendo, as informações foram obtidas a partir de 163 trabalhos nos quais encontramos 31 instituições brasileiras. Notamos, também, a pouca participação de instituições estrangeiras; somente em um trabalho foi localizado um autor ligado a instituição da França. Ao observarmos a Tabela 1, vemos as instituições com maiores ocorrências de publicação - novamente, considerando apenas a filiação institucional do primeiro autor. Quanto às instituições de maiores ocorrências, percebemos que elasse localizam na região Sudeste, especificamente, no estado de São Paulo. Isso pode se dever a um conjunto de fatores, um primeiro especificamente ligado à distribuição geográfica da ciência brasileira e um segundo, mais particular à Psicologia. No que tange ao primeiro aspecto, vemos maior concentração de instituições científicas nas regiões Sul e Sudeste (Schwartzman, 2002). Este fenômeno, inclusive, pode ter relação com a concentração de renda oriunda das estruturas socioeconômicas produzidas na República 
Velha e fortalecidas na Era Vargas. Por exemplo, além da criação das agências federais de fomento à pesquisa, o estado de São Paulo criou, em 1960, a Fundação do Amparo à Pesquisa do Estado de São Paulo (FAPESP). No que se refere particularmente à Psicologia, podemos hipotetizar, pelo menos, duas possibilidades: (1) o fato do estado de São Paulo contar com a produção de práticas e conhecimentos psicológicos, de maneira institucionalizada, desde o início do século XX. Vemos, por exemplo, a criação do Gabinete de Psicologia e Antropologia da Escola Normal de São Paulo, em 1914 (Centofanti, 2006; Tavares, 1996); e (2) o fato da cidade de São Paulo contar à época com, pelo menos, dois cursos de graduação em Psicologia, um na Faculdade de Filosofia, Ciência e Letras Sedes Sapientiae e outro na USP, o que tem relação com a produção de Psicologia institucionalizada, à época (Antunes, 2004).

Para além do circuito universitário formal (Zanelli, Borges-Andrade, \& Bastos, 2014), percebemos, com frequência menor, a publicação de autores ligados a institutos, como: Instituto de Biotipologia Criminal do Estado de São Paulo, Instituto Oswaldo Cruz, no Rio de Janeiro (RJ), Instituto de Psicologia Aplicada de Minas Gerais, em Belo Horizonte (MG); e Instituto Pieron de Psicologia Aplicada Ltda - São Paulo (SP).Tendo identificado e discutido quem publicava no "Ciência e Cultura" e suas instituições de origem, podemos direcionar a discussão para o que as pesquisas abordavam. 


\section{O que se publicava?}

\section{Tabela 2}

Frequência das áreas temáticas publicadas no período de 1949 a 1969, em ordem alfabética.

\begin{tabular}{lc}
\hline \multicolumn{1}{c}{ Área Temática } & Frequência \\
\hline Avaliação Psicológica & 44 \\
Formação em Psicologia & 16 \\
Orientação Profissional & 7 \\
Psicofarmacologia & 1 \\
Psicofisiologia & 1 \\
Psicologia da Educação & 26 \\
Psicologia do Desenvolvimento & 6 \\
Psicologia do Trabalho & 6 \\
Psicologia Experimental & 49 \\
Psicologia Social & 15 \\
$\quad$ Total & 171 \\
\hline
\end{tabular}

Após leitura e análise dos materiais, eles foram classificados de acordo com as subáreas de conhecimento da Psicologia, tendo em vista o assunto que abordava o material, o que resultou em dez áreas temáticas. É importante destacar que, em nossa análise, as classificações foram excludentes, ou seja, os materiais foram classificados somente em uma área de conhecimento. A Tabela 2 apresenta a frequência dos materiais sobre Psicologia publicados entre 1949 e 1969 no "Ciência e Cultura", por categoria. Os dados indicam uma diversidade de temáticas, mas com concentração em cinco delas, a saber: Psicologia Experimental $(n=49)$, Avaliação Psicológica $(n=44)$, Psicologia da Educação $(n=26)$, Formação em Psicologia $(n=16)$ e Psicologia Social $(n=15)$. Podemos hipotetizar que a frequência das três primeiras categorias pode se dever à história da Psicologia no Brasil na primeira metade do século XX, i.e., sua institucionalização pela instalação de laboratórios de Psicologia Experimental que frequentemente trabalhavam com parâmetros psicométricos e no contexto educacional. Além disso, a Psicologia Experimental e a Avaliação Psicológica poderiam se apresentar, em um contexto geral da ciência brasileira, à época, como uma psicologia científica, por estarem embasadas em parâmetros calcados em controle de variáveis, aparelhos de laboratório e medidas psicométricas. 
No Brasil, a Avaliação Psicológica apresentou, entre 1924 e 1947, um ritmo intenso de estudos e pesquisas, acompanhando o desenvolvimento internacional na área (Gomes, 2004). Os instrumentos internacionais chegavam ao país e eram adaptados atendendo aos preceitos psicométricos e culturais vigentes. No período analisado, observamos que os relatos apresentam testes recém traduzidos e adaptados, e suas aplicações em processos de recrutamento e seleção de pessoal e de orientação profissional. Tais dados aproximam-se dos descritos por Cruz (2002) e Zanelli e Bastos (2014); além disto, os dados podem indicar a principal identidade do psicólogo no período analisado, como postulam Hoffmann e Cruz (2003). Quanto às instituições de origem dos trabalhos, identificamos um predomínio da USP, representada por Odette Lourenção van Kolck e Theo van Kolck, os autores com maior ocorrência.

O período anterior à Lei n. 4119 foi marcado pelo desenvolvimento de conhecimento especializado e conquistas de mercado para a profissão (Pereira \& Pereira Neto, 2003), tendo início a elaboração de anteprojetos para a regulamentação da profissão. Quanto à formação profissional em Psicologia, os materiais analisados indicam uma discussão dos métodos e técnicas, objetos de trabalho e campos de atuação privativos do psicólogo, o que se aproxima das análises feitas por outros estudos (cf. Mota, Castro Neto, \& Miranda, 2016; Mota \& Miranda, 2017). A temática de Formação em Psicologia, no período analisado, foi debatida por profissionais que estiveram ligados ao processo de regulamentação da profissão em nosso país, destacando-se Annita de Castilho e Marcondes Cabral e Arrigo Leonardo Angelini, ambos da USP.

Percebemos, em Psicologia da Educação, uma preocupação sobre o processo de aprendizagem (Angelini, 1953) e sobre como proporcionar o processo de inclusão escolar, cujo enfoque recaía em crianças com desenvolvimento atípico, além de se apresentar contribuições de teorias psicológicas para uma educação mais efetiva. Também foi possível observarmos esforços dos atores ligados à Psicologia e à Educação no que tange às relações desses conhecimentos com métodos estatísticos. Ao longo do recorte temporal deste estudo (1949-1969), salientamos uma adaptação da Escala de Inteligência de Binet e Simon, “... com a finalidade pedagógica de avaliar a inteligência de crianças atrasadas na escola" (Memória, 1965, p. 61) e a preocupação dos psicólogos em criar padrões e normas para os instrumentos em questão. Quanto às filiações institucionais, destacamos, pela quantidade de material analisado, a USP nas figuras de Samuel Pfromm Netto e Nelson Rosamilha.

De 1949 a 1961, os trabalhos analisados em Psicologia Experimental, foram oriundos de diferentes modalidades de laboratórios e teorias. A partir de 1961, ano da chegada de Fred 
Keller na USP (Matos, 1996, Guilhardi, \& Madi, 1996; Todorov \& Hanna, 2010), percebe-se que os textos passam a apresentar o método experimental associada à perspectiva analíticocomportamental. Tal constatação pode indicar o período inicial do movimento em que Psicologia Experimental se transformou em uma espécie de sinônimo de Análise do Comportamento (Matos, 1998; Miranda, 2010). Com base nos documentos analisados, a USP pode ser apontada como a principal instituição de produção de conhecimento em Psicologia Experimental no período analisado, destacando-se os professores Geraldina Porto Witter, Samuel Pfromm Netto e Nelson Rosamilha como os principais autores do período.

Podemos observar que, em relação à Psicologia Social, os materiais analisados enfatizam aspectos psicossociais, como a adaptação de imigrantes à cultura brasileira; a criação de programas de planejamento familiar e controle de fertilidade, e a importância do contexto sociocultural da pessoa com paralisia cerebral. Os textos também enfatizam questões como a avaliação de atitudes de jovens frente à masculinidade e feminilidade, e os possíveis significados conotativos atribuídos aos meios de comunicação em massa. Como ocorreu nas áreas apresentadas anteriormente, em Psicologia Social as publicações também eram oriundas, principalmente, da USP. Os dados corroboram a postulação de Ferreira (2010), segundo a qual a Psicologia Social praticada na América Latina, até a década de 1970, esteve grandemente influenciada pelo paradigma da Psicologia Social Psicológica. Outro dado interessante, dentro do recorte temporal desta pesquisa (1949-1969), é o tempo transcorrido entre a publicação de trabalhos sobre Psicologia Social: um em 1953, e o próximo somente no ano de 1962, o que pode indicar o início da institucionalização da área.

\section{Considerações Finais}

Este artigo teve por objetivo descrever e analisar produções vinculadas à Psicologia, veiculadas no periódico "Ciência e Cultura", no entorno temporal da regulamentação da Lei n. 4.119 (1949-1969). A hipótese inicial era que, ao mapear tais elementos, poderíamos compreender aquilo que era produzido no país sobre a Psicologia, e que aparecia em um influente vetor de circulação da ciência brasileira no período. Observar tais aspectos nos auxilia, ainda, a compreender as articulações do conhecimento psicológico com o cenário ampliado da ciência nacional, à época. Além disso, tais elementos nos permitiram compreender a produção dos atores envolvidos com o debate legal da institucionalização do campo no Brasil. 
O periódico "Ciência e Cultura" possui uma seção de Psicologia desde seu primeiro número (1949), sendo possível observar que, desde o início, as publicações sobre esta área são constantes e que, após a Lei n. 4.119, há um aumento no número de publicações. Entre os autores, identificamos uma leve predominância do gênero feminino, devendo ser destacado o papel destas pesquisadoras como divulgadoras de conhecimento da Psicologia no período analisado. Quanto às vinculações institucionais, verificamos uma alta incidência de materiais produzidos na região Sudeste com nítida prevalência da USP. Devemos lembrar que, ainda hoje, nesta região concentram-se grande número dos centros formadores e profissionais de Psicologia.

Observamos, com a pesquisa, que no "Ciência e Cultura" inicialmente se discutia sobre formação profissional em Psicologia e Psicologia Experimental, sendo esta última constituída por diferentes modalidades de laboratórios e teorias. Foi possível identificar que, após 1961, a discussão sobre Psicologia Experimental passa a basear-se na teoria da Análise do Comportamento. Após a regulamentação da Lei n. 4.119, há uma diminuição na discussão sobre a formação profissional, e um crescimento de produção sobre Avaliação Psicológica, o que pode ser motivado por esta se constituir em função privativa da psicóloga; entretanto, tal análise foge do nosso escopo.

Neste artigo, constatamos que o número de textos sobre Psicologia publicados no "Ciência e Cultura" é muito menor que o número de resumos científicos. A publicação destes decorre da publicação dos anais dos encontros anuais da SBPC, e indicam o periódico como um veículo de divulgação científica. Ademais, tal dado pode sugerir que os atores envolvidos com a Psicologia utilizavam outros periódicos para divulgação, como os já citados Boletim de Psicologia e Arquivos Brasileiros de Psicotécnica, além de periódicos em outras áreas de conhecimento.

Acreditamos ser importante apontar algumas limitações metodológicas da pesquisa, como ter se baseado em apenas um periódico científico brasileiro e utilizar um dado recorte temporal. Desta maneira, as conclusões apresentadas não podem ser generalizadas para todo o cenário científico ou psicológico no país. Observamos que os critérios de inclusão e exclusão das fontes primárias podem ter nos levado a desconsiderar outros materiais que auxiliariam na compreensão sobre aquilo que era produzido no país sobre Psicologia. Novos estudos precisam e podem ser realizados. Apresentamos, anteriormente, a proposta de comparação dos dados da "Ciência e Cultura" com outros periódicos que veiculavam conhecimentos psicológicos. Ademais, sugerimos a discussão sobre o que motiva o impulso da divulgação de pesquisas sobre Avaliação Psicológica no período analisado. Sugere-se, também, que outros 
recortes temporais sejam considerados. Por fim, uma análise categorial dos materiais ali publicados poderia ser feita a partir de critérios hodiernos, tais como os da Coordenação de Aperfeiçoamento de Pessoal de Nível Superior (CAPES). Apesar de tais limitações, nos foi possível identificar e analisar o que era produzido no país sobre Psicologia, e que aparecia em um influente vetor de circulação da ciência brasileira, no período.

\section{Referências}

Angelini, A. L. (1953). Uma dedução teórica, explicativa, da curva de posição serial. Ciência e Cultura, 5(2), 87-92. Recuperado de http://memoria.bn.br/DocReader/docreader.aspx?bib=003069\&pesq=Uma\%20dedu\% C3\%A7\%C3\%A3o\%20te\%C3\%B3rica,\%20explicativa,\%20da\%20curva\%20de\%20p osi\%C3\%A7\%C3\%A3o\%20serial

Antunes, M. A. M. (2004). A psicologia no Brasil no século XX: Desenvolvimento científico e profissional. In M. Massimi \& M. C. Guedes (Orgs.), História da Psicologia no Brasil: novos estudos (pp.109-152). São Paulo: Cortez.

Ariès, P. (2005). A História das Mentalidades. In J. Le Goff (Org.), A História Nova (5ª ed., pp. 205-236). São Paulo: Martins Fontes. (Obra original publicada em 1978)

Baptista, M. T. D. S. (2009). Ideias divulgadas em São Paulo durante o processo histórico da regulamentação da profissão de psicólogo. Temas em Psicologia, 17(1), 119-134. Recuperado de http://pepsic.bvsalud.org/scielo.php?script=sci_arttext\&pid=S1413$389 X 2009000100011 \& \operatorname{lng}=\mathrm{pt} \& \operatorname{tlng}=\mathrm{pt}$

Boccato, I. C. (2004). Odette Lourenção Van Kolck. Avaliação Psicológica, 3(1), 69-71. Recuperado de http://pepsic.bvsalud.org/scielo.php?script=sci_arttext\&pid=S1677$04712004000100008 \& \operatorname{lng}=\mathrm{pt} \& \operatorname{lng}=\mathrm{pt}$

Brasil (1932). Decreto $n^{o}$ 21.173, de 19 de março de 1932. Converte o atual Laboratório de Psicologia da Colônia de Psicopatas, no Engenho de Dentro, em Instituto de Psicologia. Brasília, DF: Diário Oficial da União. Recuperado de http://legis.senado.gov.br/norma/440442/publicacao/15800164

Brasil (1962). Lei n. 4.119, de 27 de agosto de 1962. Dispõe sôbre os cursos de formação em psicologia e regulamenta a profissão de psicólogo. Brasília, DF: Presidência da República. Recuperado de http://www.planalto.gov.br/ccivil_03/leis/19501969/14119.htm 
Brasil (2013). Lei n. 12.842, de 10 de julho de 2013. Dispõe sobre o exercício da Medicina. Brasília, DF: Presidência da República. Recuperado de http://www.planalto.gov.br/ccivil_03/_ato2011-2014/2013/lei/L12842.htm

Brock, A. C. (2015). Presentism and diversity in the history of Psychology. Psychological Studies, 60, 373-379. doi: 10.1007/s12646-015-0315-9

Buchanan, R. D. (2003). Legislative warriors: American psychiatrists, psychologists, and competing claims over psychotherapy in the 1950s. Journal of the History of the Behavioral Sciences, 39(3), 225-249. doi: 10.1002/jhbs.10113

Campos, R. H. F. (Org.). (2001). Dicionário biográfico da Psicologia no Brasil. Rio de Janeiro: Imago.

Castro, A. C., \& Alcântara, E. S. (2011). Associação Brasileira de Psicologia Aplicada. In A. M. Jacó-Vilela (Org.), Dicionário Histórico de Instituições da Psicologia no Brasil (pp. 47-49). Rio de Janeiro: Imago.

Centofanti, R. (1982). Radecki e a psicologia no Brasil. Psicologia, ciência e profissão, 3(1), 2-50. doi: 10.1590/S1414-98931982000100001

Centofanti, R. (2006). Os laboratórios de psicologia nas escolas normais de São Paulo: O despertar da psicometria. Psicologia da Educação, (22), 31-52. Recuperado de http://pepsic.bvsalud.org/scielo.php?script=sci_arttext\&pid=S1414$69752006000100003 \& \operatorname{lng}=p t \& t \operatorname{lng}=p t$

Cintrão Forghieri, Y. (2011). Aniela Meyer Ginsberg (*02/10/1902 - + 03/08/1986) inolvidável pesquisadora, antecessora da Cad. $\mathrm{N}^{\circ}$ 11. Boletim Academia Paulista de Psicologia, 31(81), 351-357. Recuperado de https://www.redalyc.org/articulo.oa?id=946/94622764006

Cruz, M. R. (2002). Medidas psicológicas em Psicologia do trabalho e das organizações. In R. M. Alchieri, J. C. Cruz, \& J. J. Sardá Jr. (Org.), Avaliação e medidas psicológicas (pp. 173-182). São Paulo: Casa do Psicólogo.

Cunha, R. R. T., \& Santos, A. O. (2014). Aniela Meyer Ginsberg e os estudos de raça/etnia e intercultura no Brasil. Psicologia USP, 25(3), 317-329. doi: 10.1590/0103656420130013

Dantes, M. A. M. (2001). (Org.). Espaços da ciência no Brasil: 1800-1930. Rio de Janeiro: Fiocruz.

Danziger, K. (1985). Towards a conceptual framework for a critical history of psychology. In H. Carpintero, \& J. M. Peiro (Orgs.), Psychology in its historical context: Essays in 
honour of J. Brožek (pp. 99-107). Valencia: Monografias de la Revista de Historia de la Psicologia.

Fausto, B. (2003). História do Brasil (11a ed.). São Paulo: EdUSP. (Obra original publicada em 1994)

Fernandez, I. T., Polanco, F., Pereira, S. T., Beria, J. S., \& Zapico, M. G. (2017). Celebrating 50 years of the Revista Interamericana de Psicologia/Interamerican Journal of Psychology: A content analysis. Revista Interamericana de Psicologia/Interamerican Journal of Psychology (IJP), 51(3), 320-334. Recuperado de https://www.redalyc.org/pdf/284/28455448005.pdf

Ferreira, M. C. (2010). A Psicologia Social Contemporânea: Principais tendências e perspectivas nacionais e internacionais. Psicologia: Teoria e Pesquisa, 26(esp.), 5164. Recuperado de http://www.scielo.br/pdf/ptp/v26nspe/a05v26ns.pdf

Gomes, W. B. (2004). Avaliação psicológica no Brasil: Tests de Medeiros e Albuquerque. Avaliação Psicológica, 3(1), 59-68. Recuperado de http://pepsic.bvsalud.org/scielo.php?script=sci_arttext\&pid=S1677$04712004000100007 \& \operatorname{lng}=\mathrm{pt} \& \operatorname{tlng}=\mathrm{pt}$

Guilhardi, H., \& Madi, M. B. B. P. (1996). Professor Keller disse sim... Psicologia: Teoria e Pesquisa, 12(2), 113-114. Recuperado de https://www.periodicos.unb.br/index.php/revistaptp/article/view/17273

Hoffmann, M. H., \& Cruz, R. M. (2003). Síntese histórica da Psicologia do trânsito no Brasil. In M. H. Hoffmann, R. M. Cruz, \& J. C. Alchieri (Orgs.), Comportamento humano no trânsito (pp. 405-412). São Paulo: Casa do Psicólogo.

Jacó-Vilela, A. M., Mello, D. S., Ferreira, L. S. S., Messias, M. C., \& Lucas, W. S. J. (2009). Mulher, Educação e Psicologia. Anais de resumos e trabalhos completos do XV Encontro Nacional da ABRAPSO, 2009, São Paulo, SP, Brasil. Recuperado de http://www.abrapso.org.br/siteprincipal/images/Anais_XVENABRAPSO/289.\%20mul her\%2C\%20educa\%C7\%C3o\%20e\%20psicologia.pdf

Jansz, J., \& van Drunen, P. (2004). A social history of Psychology. Oxford: Blackwell Publishing.

Klappenbach, H. (2000). El titulo profesional de psicologo en Argentina: Antecedentes historicos y situación actual. Revista Latinoamericana de Psicología, 32(3), 419-446. Recuperado de https://www.redalyc.org/pdf/805/80532301.pdf 
Klappenbach, H. (2017). Los aportes de la sociobibliometria a la historia de las disciplinas científicas. Revista Guillermo de Ockham, 15(2), 5-11. Recuperado de https://pdfs.semanticscholar.org/dc6f/bb435abe85c0126c9bee6bb60716aeae0f56.pdf

Lomonaco, J. F. B. (2013). Homenagem a Samuel Pfromm Netto. Psicologia Escolar e Educacional, 17(2), 363-364. doi: 10.1590/S1413-85572013000200019

Lomonaco, J. F. B. (2014). Prof ${ }^{a}$ Geraldina Porto Witter. Boletim de Psicologia, 64(141), 217-224.

Recuperado

de

http://pepsic.bvsalud.org/scielo.php?script=sci_arttext\&pid=S0006-

$59432014000200011 \& \operatorname{lng}=p t \& t \operatorname{lng}=p t$

Machado, M. H. (Org.). (1997). Os médicos no Brasil: Um retrato da realidade. Rio de Janeiro: Fiocruz.

Matos, M.A. (1998). Contingências para a análise comportamental no Brasil. Psicologia USP, 9(1), 107-111. doi: 10.1590/S0103-65641998000100014

Matos, M. A. (1998). Carolina Bori: A psicologia brasileira como missão. Psicologia USP, 9(1), 67-70. doi: 10.1590/psicousp.v9i1.107739

Memória, J. M. P. (1965). Aplicações dos métodos estatísticos à Psicologia e à Pedagogia. Ciência $\quad e \quad$ Cultura, 17(1), 60-66. Recuperado de http://memoria.bn.br/DocReader/DocReader.aspx $? \mathrm{bib}=003069 \&$ PagFis=4858\&Pesq= Aplica\%c3\%a7\%c3\%b5es\%20dos\%20m\%c3\%a9todos\%20estat\%c3\%adsticos\%20\%c $3 \% \mathrm{a} 0 \% 20 \mathrm{Psicologia} \% 20 \mathrm{e} \% 20 \% \mathrm{c} 3 \% \mathrm{a} 0 \% 20 \mathrm{Pedagogia}$

Millán, J. D., Polanco, F., Ossa, J. C., Béria, J. S., \& Cudina, J. N. (2017). La cienciometría, su método y su filosofia: Reflexiones epistémicas de sus alcances em el siglo XXI. Revista científica Guillermo de Ockham, 15(2), 17-27. doi: 10.21500/22563202.3492

Miranda, R. L. (2010). Laboratório de Análise do Comportamento no Brasil: Percursos na UFMG na década de 1970 (Dissertação de Mestrado). Universidade Federal de Minas Gerais, Faculdade de Educação, Belo Horizonte, MG. Recuperado de https://repositorio.ufmg.br/bitstream/1843/BUOS-

8E3KBS/1/dissertacao_rodrigo_miranda.pdf

Montero, I., \& León, O G. (2007). A guide for naming research studies in Psychology International. Journal of Clinical and Health Psychology, 7(3), 847-862. Recuperado de https://www.redalyc.org/pdf/337/33770318.pdf

Mota, A. M. G. F. (2017). Desvelando controvérsias: "Problemas de ajustamento" e "Saúde Mental" nos Arquivos Brasileiros de Psicotécnica (1949-1968) (Dissertação de 
Mestrado). Universidade Católica Dom Bosco, Campo Grande, MS, Brasil. Recuperado de https://site.ucdb.br//public/md-dissertacoes/1021695-final.pdf

Mota, A. M. G. F., Cara, B. S., \& Miranda, R. L. (2018). História da Psicologia, por quê? Estudos e Pesquisas em Psicologia, 18(4), 1049-1067. Recuperado de https://www.epublicacoes.uerj.br/index.php/revispsi/article/view/42222/29293

Mota, A. M. D. G. F., Castro Neto, E., \& Miranda, R. L. (2016). "Problemas de Ajustamento" e "Saúde Mental": Controvérsias em torno de um objeto psicológico. In L. P. Almeida (Org.), Políticas públicas, cultura \& produções sociais. Campo Grande: Editora UCDB.

Mota, A. M. D. G. F., \& Miranda, R. L. (2017). Desvelando Estilos de Pensamento: ‘Diagnósticos`nos Arquivos Brasileiros de Psicotécnica (1949-1968). In L. Almeida, A. O. S. A. Duarte, M. F. P. Cassimiro, \& R. H. F. Campos (Orgs.), Psicologia, educação e o debate ambiental: questões históricas e contemporâneas. Belo Horizonte: CDPHA.

Pereira, F. M., \& Pereira Neto, A. (2003). O psicólogo no Brasil: Notas sobre seu processo de profissionalização. Psicologia em Estudo, 8(2), 19-27. doi: 10.1590/S141373722003000200003

Polanco, F. A., Beria, J. S., \& Klappenbach, H. (2017). Cinco décadas de la Revista Interamericana de Psicología: Un estudio socio-bibliométrico. Revista Interamericana de Psicologia/Interamerican Journal of Psychology (IJP), 51(3), 297-319. doi: 10.30849/rip/ijp.v51i3.910

Rosas, P., Rosas, A. \& Xavier, I. B. (1988). Quantos e quem somos. In Conselho Federal de Psicologia (Org.), Quem é o psicólogo brasileiro? (pp. 32-48). São Paulo: Edicon. Recuperado de http://newpsi.bvspsi.org.br/ebooks2010/en/Acervo_files/QuemPsicologoBrasileiro.pdf

Rosemberg, F. (1984). Afinal, por que somos tantas psicólogas? Psicologia: Ciência e Profissão, 4(1), 6-12. doi: 10.1590/S1414-98931984000100002

Rudá, C., Coutinho, D., \& Almeida-Filho, N. (2015). Formação em psicologia no Brasil: O período do currículo mínimo (1962-2004). Memorandum, 29, 59-85. Recuperado de http://www.fafich.ufmg.br/memorandum/wpcontent/uploads/2015/11/rudacoutinhoalmeidafilho01.pdf

Sá, D. M. (2006). A ciência como profissão: Médicos, bacharéis e cientistas no Brasil (18951935). Rio de Janeiro: Fiocruz. 
Schwarcz, L. M., \& Starling, H. M. (2015). Brasil: Uma biografia. São Paulo: Companhia das Letras.

Schwartzman, S. (2002). Um Espaço para a Ciência: A formação da comunidade científica no Brasil. Brasília, DF: Ministério de Ciência e Tecnologia. Disponível em: http://www.schwartzman.org.br/simon/spacept/espaco.htm

Sociedade Brasileira para o Progresso da Ciência [SBPC]. (1949). Ciência e Cultura, 1(1-2), 1-3. Recuperado de http://cienciaecultura.bvs.br/pdf/cic/v62nspe1/v62nspe1a01.pdf

Tavares, A. F. R. (1996). A ordem e a medida: escola e psicologia em São Paulo (1890-1930) (Dissertação de mestrado). Universidade de São Paulo, Faculdade de Educação. São Paulo, SP, Brasil. Recuperado de https://repositorio.usp.br/item/000744798

Teixeira, E. (2016). Projeto de Lei 4931/2016. Dispõe sobre o direito à modificação da orientação sexual em atenção a Dignidade Humana. Brasília, DF: Câmara dos Deputados. Recuperado de https://www.camara.leg.br/proposicoesWeb/fichadetramitacao?idProposicao=2081600

Todorov, J. C., \& Hanna, E. S. (2010). Análise do Comportamento no Brasil. Psicologia: Teoria e Pesquisa, 26(spe.), 143-153. doi: 10.1590/S0102-37722010000500013

Xavier, M. V. S., \& Miranda, R. L. (2018). Explorando conhecimentos e práticas psicológicas nos Arquivos de Neuropsiquiatria (1943-1949). Revista Sulamericana de Psicologia, 6(2), 261-285. Recuperado de http://ediciones.ucsh.cl/ojs/index.php/RSAP/article/view/1843

Witter, G. P. (1998). Entrevista com o Prof. Dr. Arrigo Leonardo Angelini. Psicologia Escolar e Educacional, 2(1), 55-62. doi: 10.1590/S1413-85571998000100007

Zanelli, J. C., \& Bastos, A. V. B. (2014). Inserção Profissional do Psicólogo em Organizações e no Trabalho. In J. C. Zanelli, J. E. Borges-Andrade, \& A. V. Bittencourt (Orgs.), Psicologia, Organizações e Trabalho no Brasil (2a ed., pp. 466-491). Porto Alegre: Artes Médicas.

\section{Endereço para correspondência}

Felipe Maciel dos Santos Souza

Rua Rodovia Dourados/Itahum, km 12, Um. II, Cidade Universitária, Dourados - MS, Brasil. CEP 79804-970

Endereço eletrônico: felipesouza@ufgd.edu.br

José Alberto Lechuga de Andrade Filho

Programa de Pós-Graduação em Psicologia - Curso de Mestrado Faculdade de Ciências Humanas

Avenida Costa e Silva, s/n, Campo Grande - MS, Brasil. CEP 79070-900

Endereço eletrônico: lechugadeandrade@gmail.com 


\section{Rodrigo Lopes Miranda}

Universidade Católica Dom Bosco - UCDB

Avenida Tamandaré, 6000, Jardim Seminário, Campo Grande - MS, Brasil. CEP 79117-900

Endereço eletrônico: rlmiranda@ucdb.br

Recebido em: 09/06/2019

Reformulado em: 09/08/2019

Aceito em: 01/09/2019

\section{Notas}

* Doutor em Psicologia Experimental: Análise do Comportamento.

** Mestrando em Psicologia.

*** Professor do Programa de Pós-Graduação em Psicologia da Universidade Católica Dom Bosco (UCDB).

Este artigo de revista Estudos e Pesquisas em Psicologia é licenciado sob uma Licença Creative Commons Atribuição-Não Comercial 3.0 Não Adaptada. 